DIACRONIE

\section{Diacronie}

Studi di Storia Contemporanea

$N^{\circ} 13,1 \mid 2013$

Contrabbandieri, pirati e frontiere: per una storia delle pratiche informali nell'America Centrale (XVII-XXI secolo)

\title{
Adriano Vinale, Pragmatismo americano. Razza e democrazia
}

\section{Valerio Romitelli}

\section{OpenEdition \\ Journals}

Edizione digitale

URL: http://journals.openedition.org/diacronie/801

DOI: 10.4000/diacronie.801

ISSN: 2038-0925

Editore

Association culturelle Diacronie

Notizia bibliografica digitale

Valerio Romitelli, «Adriano Vinale, Pragmatismo americano. Razza e democrazia », Diacronie [Online], N

13, 1 | 2013, documento 18, Messo online il 01 avril 2013, consultato il 22 septembre 2020. URL

http://journals.openedition.org/diacronie/801 ; DOI : https://doi.org/10.4000/diacronie.801 


\section{8/}

\section{RECENSIONE:}

\section{Adriano VINALE, Pragmatismo americano. Razza e democrazia, Napoli, Cronopio, 2012, 261 pp.}

a cura di Valerio ROMITELLI*

Come gli ultimi accordi finanziari confermano, sembra proprio che la Cina stia riuscendo a sopravanzare e mettere al traino la potenza economica americana.

In senso contrario va, però, la strategia Nato orientata a stringere i ranghi tra gli alleati pur di non recedere in quella sorta di prolungamento della guerra fredda che continua a trattare Mosca e Pechino come nemici giurati.

D'altra parte, se ci si chiede quale sia la scuola di pensiero che ha indirizzato la più recente e spettacolare crescita delle stesse Russia e Cina, oltre che degli altri paesi emergenti, quali India e Brasile, la risposta deve essere cercata dalle parti di quel neoliberismo di cui gli Stati Uniti, assieme alla Gran Bretagna, sono terra d'origine e di tutt'ora persistente coltivazione. Non altra è la fonte prima cui da più di trent'anni si abbevera tutto quel managerialismo globale i cui meriti diventano sempre meno paragonabili alle crescenti colpe. Da quando il comunismo ha cominciato a perdere sempre più pezzi e fascino, mentre gli Stati Uniti si avviavano verso una completa egemonia mondiale, le nuove forme di governance all'insegna di "meno Stato più mercato" hanno sì dimostrato qualche virtù, ma anche vizi ben superiori. Infatti, non vi è stata solo una meno iniqua redistribuzione della ricchezza globale, a favore di paesi già poveri ( come appunto quelli del "BRIC"). Vi sono stati anche"effetti collaterali" tutt'ora perduranti e sempre meno sopportabili: una progressiva crescita dell' ingiustizia sociale all'interno di ogni paese si è accompagnata all'emarginazione di più di un miliardo di popolazione sotto il livello della sopravvivenza, mentre l'economia globale subiva un'esplosione della finanza senza precedenti, la quale, dopo aver portato a svariate crisi locali e contingenti, è sfociata nell'attuale, di portata illimitata e senza uscite prevedibili. 
D’altra parte, il ritorno della politica, che da più di tre anni ha cominciato ad essere invocato e riattivato tra i paesi tradizionalmente più ricchi come rimedio ai danni dei precetti neoliberisti, è ben lungi dal negarli alla radice.

Né su scala globale è facile trovare chiare alternative. Anche laddove, specie tra alcuni paesi emergenti, qualcosa in tal senso pare esserci, comunque latitano prospettive strategiche altrettanto sistematiche e generalizzabili di quella contrastata. Le uniche soluzioni paiono ridursi al contenere, rettificare o mascherare quella stessa che da più di trent'anni imperversa ovunque, presentandosi come pura e semplice conseguenza delle esigenze dei mercati.

Ciononostante, secondo narrative care anche all'amministrazione Obama, la globalizzazione in corso sarebbe già frutto di una progressiva multipolarità democratica. Ma lo stesso vasto consenso goduto da tale ben poco realistica visione dimostra quanto l'egemonia culturale americana non trovi rivali, a livello intellettuale. Se così è, allora il nostro tempo si trova in uno strano paradosso.

Quello rappresentato dal fatto che la crisi di un'“economia mondo", quella con a capo gli Usa, è in un qualche modo compensata dal mantenimento, se non dal rafforzamento, del loro ruolo egemone sia sul piano militare, sia su quello culturale.

Abbandonandosi alla tentazione di congetture storiche di "lunghissima durata", si potrebbe forse anche temere che l'attuale espansione economica della Cina, restando solo tale, dunque non aprendo alcun nuovo orizzonte né politico né culturale a livello mondiale, finisca per indurre il concorrente principale e suoi alleati a reazioni su quel piano militare che, come al tempo della Guerra dell'Oppio, potrebbe dimostrarsi sempre in grado di infrangere ogni astuzia commerciale e finanziaria.

Cosa c'entra con tutto ciò il libro di Vinale, Pragmatismo americano. Razza e democrazia, Cronopio, 2012?

Direttamente, nulla. Indirettamente, moltissimo.

In effetti, la sua ricostruzione della storia intellettuale e culturale degli Stati Uniti non va oltre l'inizio del secolo scorso. Né il rigore della sua argomentazione quanto mai documentata, stringata e fine, dà adito a qualsiasi congettura o conclusione generalista o generalizzante. Tuttavia mi pare importante invitare alla sua lettura anche chi non è un amante o uno studioso di "americanismo". Leggendo questo testo ci si può infatti convincere come la genealogia da esso ricostruita - con obbligatori richiami al metodo dell'“archeologia del sapere" praticata da Foucault - sia divenuta inevitabilmente e sempre più anche nostra genealogia.

L'egemonia culturale americana non ha certo risparmiato l'Italia, nonostante che il "volere fare l'americano", a suo tempo canzonato da Sordi e Carosone, sia oramai 
divenuto un "dover fare" tanto radicato e profondo, da non trovare più neanche satire adeguate, ma solo adesioni incondizionate. Per convincersene basterebbe notare come non ci sia istituzione della nostra Repubblica, né impresa o associazione, volontaria o meno, che non faccia proprio il linguaggio della governance all'americana. Così, nonostante il suo imperversare ormai trentennale, nonostante la sua assonanza coi dogmi neoliberisti, nonostante la crisi devastante che ha finito per favorire, tale linguaggio continua ad essere celebrato come novità risolutiva di ogni problema politico, sociale ed economico.

Ecco allora dove sta l'importanza non solo specificamente scientifica del libro di Vinale: sta nel fatto che esso permette non solo di capire vicende intellettuali passate e lontane, ma anche di capire qualcosa di fondamentale nei nostri stessi attualissimi modi di porci nei confronti del mondo.

Di cosa sto parlando è subito facilmente desumibile anche solo dalle quattro parole che compongono titolo e sottotitolo.

Il "pragmatismo" non è forse l'etichetta normalmente più usata per designare il tipo di comportamento che in Italia, come in gran parte del mondo, gode dei maggiori consensi? In tale espressione non si è forse soliti vedere una felice alternativa alle complicate anticaglie filosofiche del vecchio continente, quali idealismo o materialismo? E nel fatto che il pragmatismo sia proprio "americano" non si vede forse la conferma della sua efficienza dimostrata proprio dai successi che hanno fatto dell'economia a stelle e strisce l'economia leader del mondo? Quanto poi a "democrazia", niente sembra più certo oggi del fatto che essa rappresenti il migliore regime mai concepito dall'umanità, ossia del fatto che esso sia la più naturale conseguenza del pragmatismo e che abbia la sua patria nella stessa America.

Rispetto a questa facile ed edificante lettura del titolo di Vinale stride però la parola “razza”, tanto più accostata com'è alla parola democrazia. Probabilmente il lettore più coscientemente democratico, pragmatico e quindi ammiratore degli Usa avrebbe preferito "razze", al plurale. Così avrebbe potuto intendere che in questo testo si riprende la solita celebrazione di questo paese come melting pot di comunità tanto diverse quanto capaci di convivere le une delle altre - a parte conflitti e violenze varie. Ma non è affatto così. E il singolare del termine "razza" indica invece l'ostica durezza del fondamento stesso di tutto l'insieme di queste parole. In effetti, "primo obiettivo" del lavoro di Vinale, secondo le sue stesse parole, è «rilevare la radice razzista della democrazia americana»1.

${ }^{1}$ VINALE, Adriano, Pragmatismo e democrazia. Razza e democrazia, Napoli, Cronopio, 2012, p. 11. 
Un consuntivo finale dei risultati raggiunti in tal senso avrebbe forse giovato alla chiarezza di tutto il testo, che, invece, si arresta un po' bruscamente con la sintesi del pensiero di Dewey negli anni Venti. Ma a ben vedere, le sue frasi, commentate nelle ultime righe, suggellano al meglio tutto il percorso che questo libro ci restituisce e che ha appunto al centro la pretesa di superiorità, al fondo razziale, dell'“essere americano”.

Ciò che mostra infatti Vinale chiudendo il suo scritto, è quanto Dewey chiedeva alle Società delle Nazioni allora in corso di formazione: niente di meno che la costruzione di un'international mind ispirata proprio alla democrazia americana ${ }^{2}$. Che essa dovesse diventare un modello addirittura mentale per il mondo intero: questa dunque la pretesa di uno dei più riconosciuti padri del pragmatismo, con cui si interrompe molto significativamente il lungo e complesso percorso del libro. Quasi ad indicare, così mi è piaciuto leggerlo, che la sua portata analitica si proietta ben oltre Dewey.

Nel suo dipanarsi, tale percorso prende le mosse dal mito della supremazia genetica anglosassone e dalla sua rilevanza, a metà Ottocento, nell'epoca della dottrina Monroe e dell'egualitarismo jacksoniano, per poi concentrasi sull'importanza dell'influenza di Darwin, di Lamarck - ma soprattutto di Spencer - nei dibattiti politici e sociologici dell'America ottocentesca.

Come vi sia stato possibile concepire la democrazia quale «unità biologica»3, «forma non di governo, ma di vita»4, Vinale lo chiarisce con grande precisione tramite l'analisi di nodi concettuali reperiti nelle diverse formulazioni e recezioni dell'evoluzionismo, nonché dei rispettivi retroterra filosofici. La "variazione casuale", la "civilizzazione" come processo "dall'omogeneità alla complessità", la funzione in ciò della competizione tra uomo e uomo, ma ancor di più tra comunità e comunità, l'importanza della dimensione militare nell'evoluzione umana, la "selezione naturale" premiante l'“idoneità alla sopravvivenza”, l'opzione repubblicana come prospettiva "intensiva" dello sviluppo democratico versus l'opzione più propriamente democratica incline piuttosto ad una strategia "estensiva" (dunque espansionistica), la "credenza" come motore primo dell'“azione”: questi alcuni dei tanti nodi concettuali decisivi nei dibattiti e nelle ricerche dell'America ottocentesca che Vinale sviscera acutamente, giungendo a mostrarne anche le implicazioni sulle origini e gli sviluppi in questo paese, tanto dalla psicologia, quanto dalla semiotica. Accorte e sintetiche riletture di Hume,

\footnotetext{
2 Ibidem, p. 253.

${ }^{3}$ Ibidem, p. 15 .

${ }^{4}$ Ibidem, p. 21.
} 
come riferimento fondamentale, di Charles Sanders Peirce e William James si accostano così a quelle già menzionate di Darwin, Spencer, Lamarck e Dewey.

Ma, il punto di originalità maggiore del libro sta nella ripresa e nel commento di autori meno universalmente noti, ma di grande rilevanza per l'America del XIX secolo ( quali Bagheot, O'Sullivan, Gray, Ward, Wright ), per altro sempre trattati alla luce del contesto determinato dagli eventi maggiori, quali la Guerra di Secessione, quella contro l'Impero Spagnolo o l'intervento nel primo conflitto mondiale. Tra queste figure, ad esempio, appare quanto mai attuale quella di William Graham Summer. Convinto repubblicano, dunque conservatore e antiespansionista, questi nei primi anni Ottanta dell'Ottocento trattava infatti del rapporto tra lavoro e capitale in modi che possono ricordare il ben più recente tema del "capitale umano" quale lo ha presentato Gary Becker negli anni Novanta di questo secolo.

C'è dunque anche un'altra importante implicazione del libro di Vinale sull'attualità: che la sua lettura può giovare notevolmente alla discussione e alle ricerche intorno a ciò che si chiama solitamente "neoliberalismo". Grazie a quanto si può leggere nel testo si è infatti in grado di ragionare su alcuni punti fondamentali riguardo a questo orientamento.

Primo, che esso non è stato affatto una trovata dei "soliti” capitalisti, galvanizzati trent'anni fa dall'afflosciarsi del comunismo. Secondo, che esso si radica, invece, in una profonda tradizione culturale americana, la quale viene da molto lontano, ma che è riuscita ad imporsi nel mondo solo trent'anni fa, appunto. Terzo, che esso è tutto tranne che un puro orientamento economico, particolarmente tecnico, che si limiterebbe semplicemente a "servire i mercati". Quarto, che si tratta invece di una visione dalla pretesa ben più ampia, onnicomprensiva della vita, della morte, dell'essere e del nulla, insomma, ontologica. Quinto, che, proprio per tutte queste ragioni, non può essere considerato una qualche continuazione del liberalismo di matrice inglese, questo sì orientato soprattutto a definire termini e limiti dell'economia politica.

Dal che se ne potrebbe trarre la conclusione che l'espressione stessa di "neoliberalismo" non è quella giusta, perché, in effetti, si tratta piuttosto delle conseguenze ultime di quello che Vinale ci insegna a riconoscere come "pragmatismo". Personalmente 5 ho provato a ripensare queste conseguenze identificandole sotto l'etichetta di quell'orientamento che attualmente impazza tra tutte le più disparate scienze ( in particolare quello "neuro-"): cioè il cognitivismo. Ma certo una maggior comprensione dei suoi presupposti pragmatisti, quale Vinale rende disponibile,

5 ROMITELLI, Valerio (a cura di), Fuori della società della conoscenza: ricerche di etnografia del pensiero, Ed. Infinito, 2009, Introduzione. 
permette di distinguere meglio quanto sotto questa etichetta si nasconda di operativamente sperimentale e quanto invece di puramente celebrativo della presunta superiorità biologica della democrazia americana. 


\section{* L'autore}

Valerio Romitelli è ricercatore di Storia delle dottrine politiche nella Facoltà di Scienze politiche dell'Università di Bologna. Studioso di storia della politica italiana ed europea moderna e contemporanea, con particolare attenzione alle questioni metodologiche e d'approccio problematico, è anche ricercatore delle metodologie delle scienze sociali incentrate sulla proposta di un approccio etnografico a distanza da ogni metalinguaggio e volto a conoscere e pensare il pensiero di popolazioni a disagio a partire dalle loro stesse parole. È autore di numerosi libri tra i quali Quando si è fatto politica in Italia? Storia di situazioni pubbliche (1796-1951), Soveria Mannelli, Rubbettino, 2004; Etnografia del pensiero. Ipotesi e ricerche, Roma, Carocci editore, 2005; Fuori della società della conoscenza. Ricerche di etnografia del pensiero, Roma, Infinito Edizioni, 2009.

URL: < http://www.studistorici.com/progett/autori/\#Romitelli >

\section{Per citare questo articolo:}

ROMITELLI, Valerio, «Recensione: Adriano VINALE, Pragmatismo americano. Razza e democrazia, Napoli, Cronopio, 261 pp.», Diacronie. Studi di Storia Contemporanea: Contrabbandieri, pirati e frontiere: per una storia delle pratiche informali nell'America Centrale (XVII-XXI secolo), 29/04/2013,

URL:< http://www.studistorici.com/2012/04/29/romitelli_numero_13/ >

Diacronie Studi di Storia Contemporanea $\theta$ www.diacronie.it

Risorsa digitale indipendente a carattere storiografico. Uscita trimestrale.

redazione.diacronie@hotmail.it

Comitato di redazione: Marco Abram - Jacopo Bassi - Luca Bufarale - Alessandro Cattunar - Elisa Grandi - Deborah Paci - Fausto Pietrancosta - Matteo Tomasoni - Luca Zuccolo

Diritti: gli articoli di Diacronie. Studi di Storia Contemporanea sono pubblicati sotto licenza Creative Commons 2.5. Possono essere riprodotti a patto di non modificarne i contenuti e di non usarli per fini commerciali. La citazione di estratti è comunque sempre autorizzata, nei limiti previsti dalla legge. 